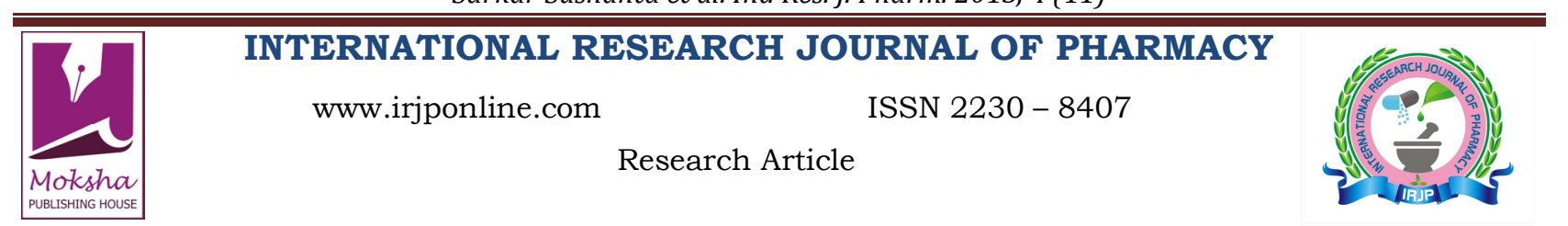

\title{
SOLUBILITY IMPROVEMENT OF POORLY WATER SOLUBLE DRUG FENOFIBRATE BY SOLID DISPERSION IN POLYETHYLENE GLYCOL-HYDROXY PROPYLE METHYLE CELLULOSE MIXTURE
}

\author{
Sarkar Sushanta ${ }^{1}$, Islam Md. Saiful ${ }^{2}$ \\ ${ }^{1}$ Department of Pharmaceutical Technology, Faculty of Pharmacy, University of Dhaka, Dhaka, Bangladesh \\ ${ }^{2}$ Department of Pharmaceutical Technology, Faculty of Pharmacy, University of Dhaka, Dhaka, Bangladesh \\ *Corresponding Author Email: sushanta_du@yahoo.com
}

Article Received on: 17/09/13 Revised on: 21/10/13 Approved for publication: 06/11/13

DOI: $10.7897 / 2230-8407.041108$

IRJP is an official publication of Moksha Publishing House. Website: www.mokshaph.com

(C) All rights reserved.

\section{ABSTRACT}

The objective of this study is to formulate solid dispersion of fenofibrate by hot melt extrusion method for improving aqueous solubility. Different formulation was prepared mixing microcrystalline drug, Poly Ethylene Glycol (PEG) and other excipient at specific ratio. The drug was dissolved in the melt of excipient at $65-70^{\circ} \mathrm{C}$, cooled below $40^{\circ} \mathrm{C}$, and grinded to prepare fine powder. The solid system was characterized by Differential Scanning Calorimetry (DSC), Scanning Electron Microscope (SEM), Fourier Transform Infrared Spectroscopy (FTIR), the dispersion testing in the water and simulated gastric fluid. It was confirmed that there is no chemical interaction between drug and excipient. In-vitro release testing showed that $76.85 \%$ of the drug dissolved in distilled water in 60 minutes. Almost $100 \%$ of drug release was found within 20 minutes and 30 minutes in $2 \%$ Sodium Lauryl Sulfate and $1 \%$ Tween- 80 simulated gastric fluid respectively for drug, PEG and HPMC ratio 1:7.5:2.5. Thus, the aqueous solubility of this poorly water-soluble drug was greatly enhanced by solid dispersion in a surface-active carrier.

Keywords: Poorly water soluble drug, solid dispersion, hot melt extrusion, Fenofibrate, Hydroxy Propyle Methyle Cellulose (HPMC).

\section{INTRODUCTION}

A poorly water-soluble drug is the one whose dissolution in the gastro intestinal (GI) fluid under ordinary conditions takes a longer time than its transition through the absorption sites in the GI tract ${ }^{2}$. Dissolution in the GI fluid is a critical requirement for a poorly water-soluble drug to be absorbed in the GI tract. When water solubility is less than $1 \mu \mathrm{g} / \mathrm{ml}$, which is often the case for contemporary drug candidates, the bioavailability from conventional tablet formulations may be unacceptable. With the recent advent of high throughput screening of potential therapeutic agents, the number of poorly soluble drug candidates has risen sharply and the formulation of poorly soluble compounds for oral delivery presents one of the most frequent and greatest challenges to formulation scientists ${ }^{5}$. The dissolution rate of poorly watersoluble drugs from crystalline formulation can be increased by reducing the particle size and increasing surface area ${ }^{10,11}$. Crystalline drug is in a stable state in the formulated product and will remain in a physically stable state throughout the dissolution phase in the gut lumen ${ }^{9}$. Many physical approaches to improve aqueous solubility include salt formation, particle size reduction, emulsions, micelles, liposome, and macro-/nano particles, but most of the approaches are liquid based which results in problems like instability, in-vivo uncertainty and manufacturing cost $^{3}$. Unlike liquid formulation techniques solid dispersion systems shows significant results in increasing aqueous solubility of poorly water-soluble drug where the drug is dispersed either as fine particles or molecularly in solid water-soluble matrices ${ }^{1}$. The solid dispersion technique is useful to reduce the particle size from crystalline to micro-crystalline level, which ultimately enhances water solubility of poorly water soluble $\operatorname{drug}^{7}$. The hot melt extrusion method draws the attention of formulation scientists due to the advantage of continuous production without any organic solvents ${ }^{4,5}$. Single or combination of carriers may also be essential for development of solid dispersion ${ }^{13}$. Different surfactants are used in solid dispersion to improve solubility of poorly water-soluble drugs ${ }^{6}$. There are only few suitable surfactants for solid dispersion ${ }^{3}$. Polyethyleneglycol (PEG) and polysorbate 80 has potential for use in solid dispersion ${ }^{8}$. In this study we used Polyethyleneglycol (PEG) and other surfactants in solid dispersion to find efficient surface-active agent combination. Finally potency of solubility enhancement of PEG and Hydroxypropylemethylecellulose (HPMC) was evaluated by improvement of water solubility of the poorly water-soluble drug fenofibrate.

\section{MATERIALS AND METHODS}

\section{Materials}

Fenofibrate micronized powder was provided by Beximco Pharmaceuticals Bd. Ltd. Dhaka, Bangladesh. Polyvinylpyrolidone (PVP) K30, Proloxomer-407, and Sodiumcarboxymethylecelloluse (Na-CMC) were provided by Shin-Etsu Chemical Co. Ltd. Tokyo, Japan. Hydroxypropylemethylecellulose $5 \mathrm{cps}$, Maize starch, Pre gelatinized starch, Tween-80, and Tween-20 were provided by BASF, Ludwigshafen, Germany.

\section{In-vitro Dissolution Study}

A dissolution study of solid dispersion powder was performed according to the USP apparatus II paddle method $\left(75 \pm 2 \mathrm{RPM}\right.$ at $\left.37 \pm 0.5^{\circ} \mathrm{C}\right)$ in water, $2 \%(0.025 \mathrm{M})$ sodiumlaurylsulphate (SLS) and $1 \%(0.015 \mathrm{M})$ Tween-80 solution. Aliquots of dissolution medium collected at different interval of time $(0,10,20,30,40,50$ and 60 minutes) were filtered through $0.45 \mu \mathrm{m}$ syringe filter.

\section{Formulation}

PEG 6000 was heated at $70^{\circ} \mathrm{C}$ until a clear liquid was formed. Then fenofibrate was added in the molten PEG followed by addition of excipient. The mixture was allowed to cool to room temperature and grinded to form fine powder. Powder solid dispersion was preserved in an air-tight screw cap vials and were kept in desiccators until further use. 
Table 1: Percent drug loading in the formulation containing drug, PEG6000 and solubility enhancer at a ratio $1: 5: 5$

\begin{tabular}{|c|l|}
\hline Excipient & \% Drug Loading* \\
\hline HPMC & $98.92 \pm 2.3$ \\
\hline Poloxamer 407 & $99.27 \pm 2.4$ \\
\hline PVP K 30 & $95.68 \pm 1.7$ \\
\hline Tween 20 & $97.96 \pm 2.1$ \\
\hline Tween 80 & $95.32 \pm 1.8$ \\
\hline Maize Starch & $96.90 \pm 1.9$ \\
\hline egelatinized Starch & $90.13 \pm 1.6$ \\
\hline Sodium CMC & $70.32 \pm 1.9$ \\
\hline \multicolumn{2}{|c|}{ *Mean \pm SD $(n=6)$} \\
\hline
\end{tabular}

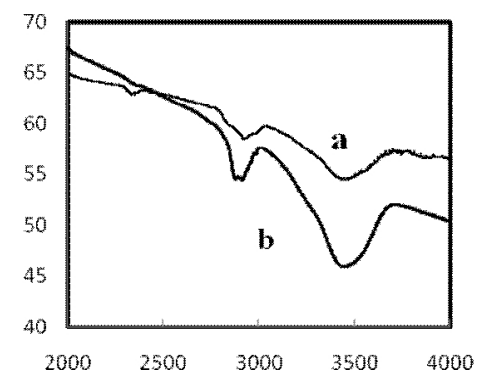

Figure 1: FTIR study of pure drug (a) and solid dispersion powder (b)
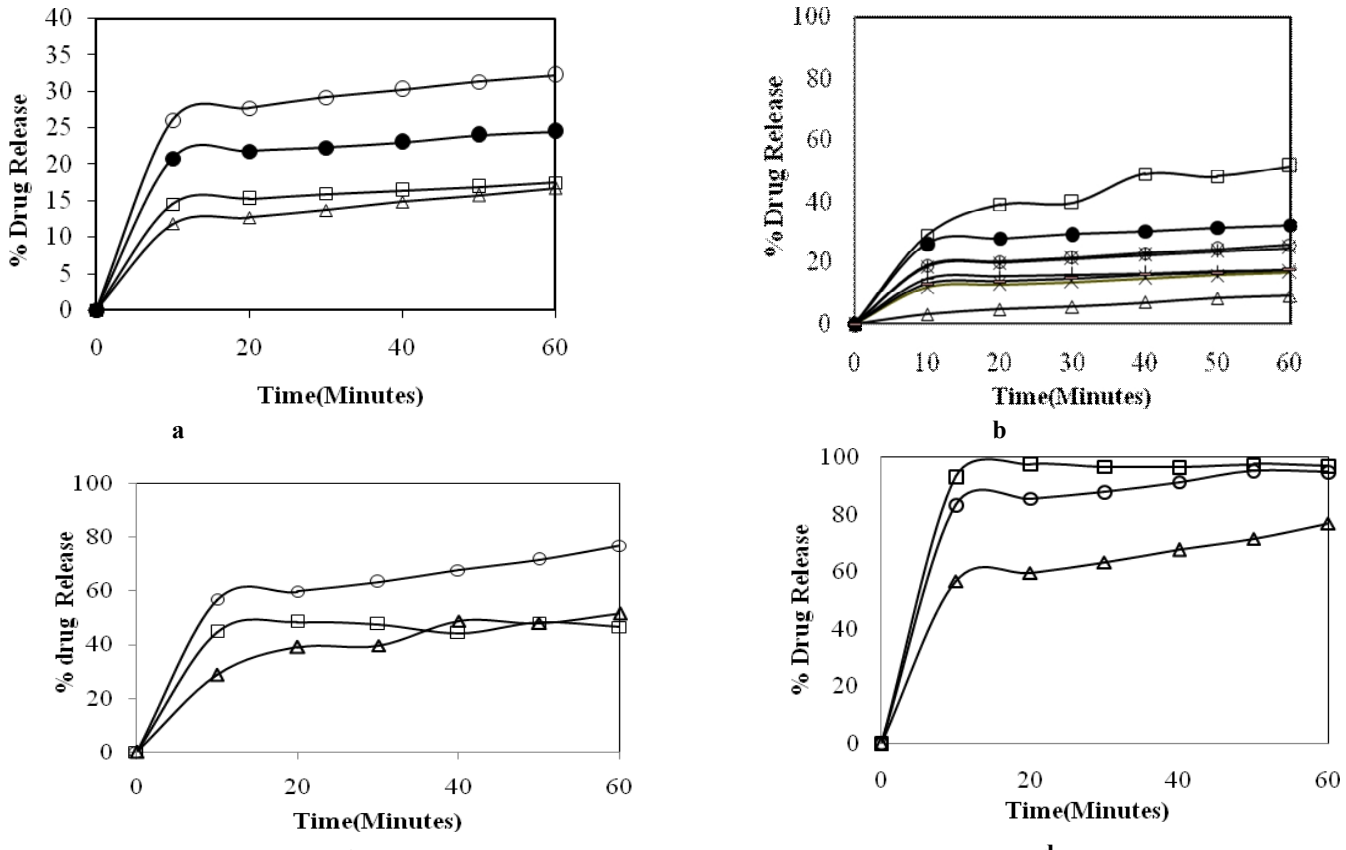

d

Figure 2: Dissolution of drug from solid dispersion with PEG-6000 and solubility enhancer at different ratio in $1000 \mathrm{~mL}$ of water at $37^{\circ} \mathrm{C}$ and at 100 rpm. (a) Key to ratio of drug and PEG-6000 ( $\Delta-1: 1,-1: 10, \bullet-1: 4,0-1: 5)$. (b) Drug, PEG-6000 and solubility enhancer ratio 1:5:5 ( $\Delta$ Polyvinylpyrolidone, HPMC, • Proloxomer-407, o Maize starch, *Tween-80, + Pregelatinized starch, $\times$ Tween-20, - Sodium Carboxymethyle celloluse) (c) Drug, PEG-6000 and HPMC ratio ( 1:3.5:1.5, $\Delta$ 1:5:5, o 1:7.5:3.5). (d) Comparative dissolution study of solid dispersion powder (Drug, PEG-6000 and HPMC 5cps ratio 1:7.5:3.5) in water and simulated gastric fluid ( $0.025 M$ sodium lauryl sulphate(SLS), $\Delta$ 0.015 M Tween-80, o distilled water). Each datum point represents average of three determinations
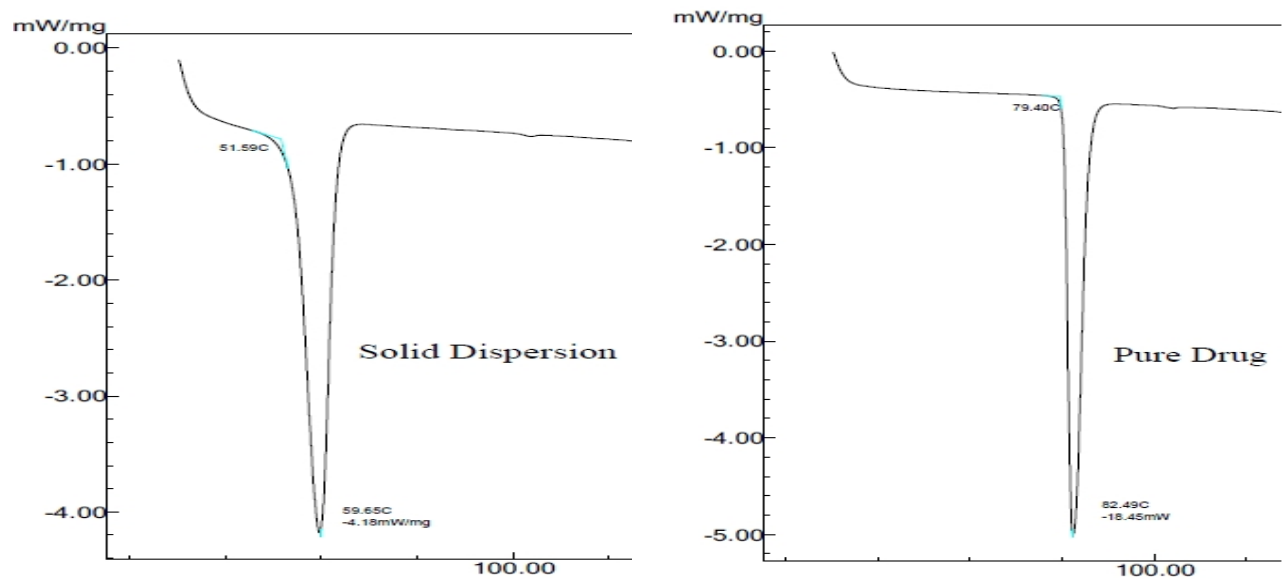

Figure 3: DSC scans of pure drug and solid dispersion containing drug, PEG-6000 and HPMC 

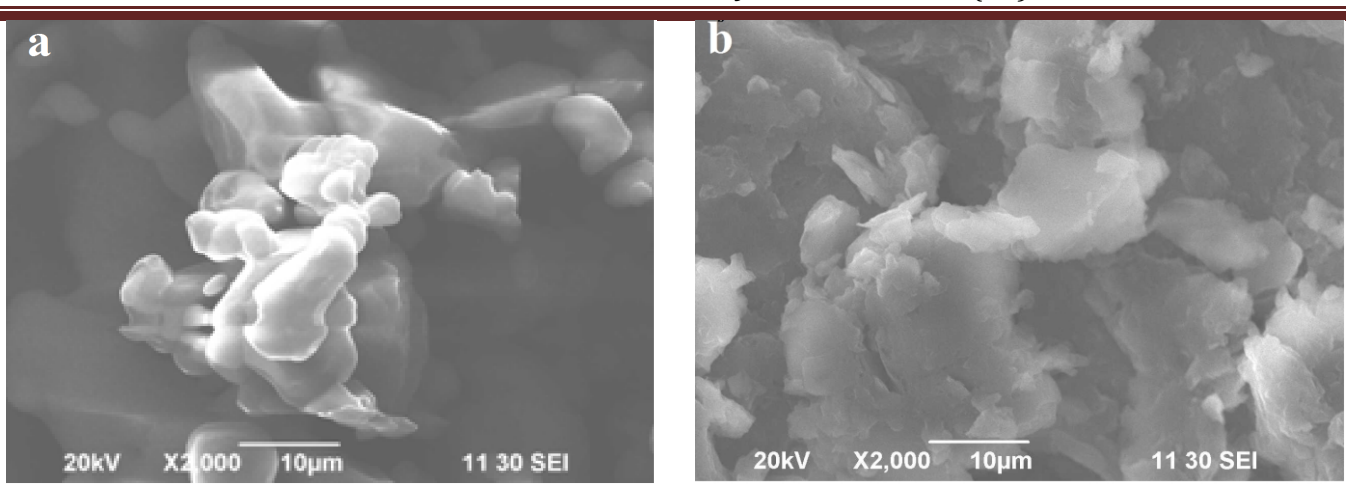

Figure 4: Scanning electron microscopy image of (a) pure drug and (b) solid dispersion containing drug, PEG-6000 and HPMC

\section{Differential Scanning Calorimetry (DSC)}

The DSC study was carried out using DSC-60, Shimadzu Corp. Kyoto, Japan. The instrument is comprised of calorimeter (DSC-60), flow controller (FCL-60), thermal analysis (TA-60) and operating software (60). Sample (3.70 $\mathrm{mg}$ ) was heated in sealed aluminum pans under nitrogen flow $\left(20 \mathrm{ml} / \mathrm{min}\right.$ ) with a control scanning rate of $10^{\circ} \mathrm{C} / \mathrm{min}$ from $30^{\circ} \mathrm{C}$ to $300^{\circ} \mathrm{C}$. An empty aluminum pan was used as reference.

\section{Scanning Electron Microscopy (SEM)}

The shape and surface morphology of pure Fenofibrate and solid dispersions were studied by scanning electronic microscopy (SEM-GEOL JSM-6490LA). The samples were mounted on double-sided adhesive tape that had previously been secured on aluminum stubs and then analyzed. The acceleration voltage was $20 \mathrm{KV}$. The samples were analyzed at magnifications of $500 \mathrm{X}, 1000 \mathrm{X}, 2000 \mathrm{X}$ and $9000 \mathrm{X}$.

\section{Fourier Transform Infrared Spectroscopy (FTIR)}

The IR spectra of each sample were recorded using an FTIR spectrophotometer (FT-IR 8400S, Shimadzu). The samples were scanned over the frequency range $4000 \mathrm{~cm}^{-1}-400$ $\mathrm{cm}^{-1}$.

\section{RESULTS AND DISCUSSION \\ In-vitro Dissolution}

Micronized drug particle were mixed with PEG-6000 at different ratios $(1: 1,1: 4,1: 5$ and $1: 10)$ to form a binary solid dispersion. The drug and carrier ratio 1:5 was selected for ternary solid dispersion formulation as per dissolution data shown in Figure - 2a. Further formulation was performed with the drug and PEG-6000 at a ratio of 1:5 and another solubility enhancer added to the same amount of PEG-6000 (Table 1). From 8 solubility enhancers drug, PEG-6000 and HPMC 5 cps ratio (1:5:5) was chosen for further study as per dissolution data shown in Figure 2b. More solid dispersion formulations were developed by changing the relative amounts $25 \%(\mathrm{w} / \mathrm{w}), 30 \%(\mathrm{w} / \mathrm{w})$, and $50 \%(\mathrm{w} / \mathrm{w}) \mathrm{HPMC}$ of PEG-6000. Dissolution of solid dispersion was performed in $1000 \mathrm{~mL}$ of water at $37^{\circ} \mathrm{C}$, where the aliquots were analyzed by UV-visible spectroscopy after filtering through a $0.45 \mu \mathrm{m}$ syringe filter. The primary dissolution study indicates (Figure 2c) $56.78 \%$ drug released from solid dispersion containing $50 \%(\mathrm{w} / \mathrm{w})$ HPMC of PEG-6000. Surfactant was added separately in water to create sink condition and simulate gastric fluid, which does not necessarily reflect in-vivo performance of solid dispersion. Almost $93 \%$, and $83 \%$ drug was dissolved from solid dispersion containing $50 \%$ (w/w) HPMC of PEG in $2 \%$ sodium lauryl sulphate (SLS) and $1 \%$ Tween- 80 solutions respectively in 60 minutes (Figure 2d). It was observed that a plateau was reached after $56.78 \%$ drug released within 10 minutes in water and maximum $76.85 \%$ drug release obtained after 60 minutes. Milky color in the dissolution medium indicated the dispersion of active material as fine particles.

\section{Solid State Characterization}

Fourier Transform Infrared Spectroscopy

The Fenofibrate spectra showed sharp characteristic peaks at $1731.14 \mathrm{~cm}^{-}{ }^{-}$(R-O-R, Ester), $2983.93 \mathrm{~cm}^{-}{ }^{1}$ and $2935.71 \mathrm{~cm}^{-}{ }^{1}$ (Benzene ring), $1650.3 \mathrm{~cm}^{-}(\mathrm{C}=\mathrm{O}$, carbonyl) (Figure 1). The above characteristic peaks appear in the spectra of TSD powder at the same wave number showing no interaction among the excipient, carrier and drug.

\section{Differential Scanning Calorimetry (DSC)}

Thermogram of pure fenofibrate exhibited an intense peak at $82.49^{\circ} \mathrm{C}$ indicating the melting point of the drug and its crystalline nature (Figure 3). In the thermogram of formulations solid dispersion showed a similar peak, which indicated the presence of the drug in crystal form. However, the intensity of the peak of solid dispersion was lowered, indicating a lower crystalline nature of the drug. In addition, entrapment of drug crystals inside the carrier matrix may also be attributed to the appearance of a blunt peak. The characteristic melting peak broadened and shifted slightly with reduced intensity for solid dispersion. This may be attributed to high polymer concentration and uniform distribution of the drug in the crust of the polymer, resulting in complete miscibility of molten drug in the polymer.

\section{Scanning Electron Microscopy Study}

Fenofibrate exists in fine regular particulate shape in the range of particle size 10-15 $\mu \mathrm{m}$ range that shown in Figure 4a. The SEM result showed that fine regular shaped particles of pure drug were dispersed within the carrier matrices, indicating that the particles of the drug are incorporated within the carrier system. The surface morphology studies revealed that the solid dispersions were closely compacted into small irregular forms. The original morphology of components disappeared, which supported DSC.

\section{CONCLUSION}

The dissolution property of hot-melt extruded solid dispersion of poorly water-soluble drug can be enhanced by adding polymer, which can meet specific requirements of drug dispersion ${ }^{12}$. Polymers and surfactants blend are used to improve dissolution property of poorly water-soluble drug as the blends are stable against re-crystallization of solid 
dispersions ${ }^{14}$. Our study found improved solubility and dissolution of fenofibrate from solid dispersion of PEG-6000 and HPMC by hot-melt extrusion.

\section{REFERENCES}

1. Chiou WL, Riegleman S. Pharmaceutical application of solid dispersion systems. J Pharm Sci 1971; 60(9): 1281-1302. http://dx.doi.org /10.1002/jps.2600600902 PMid:4935981

2. Horter D, Dressman JB. Influence of physicochemical properties on dissolution of drugs in the gastrointestinal tract. Adv Drug Deliv Rev 1997; 25(1): 3-14. http://dx.doi.org/10.1016/S0169-409X(96)00487-5

3. Serajuddin ATM. Solid dispersion of poorly water-soluble drugs: early promises, subsequent problems and recent breakthroughs. J Pharm Sci 1999; 88(10): 1058-1066. http://dx.doi.org/10.1021/js9804031 PMid: 10514356

4. Leunar C, Dressman J. Improving drug solubility for oral solubility using solid dispersions. Eur J Pharm Biopharm 2000; 50(1): 47-60. http://dx.doi.org/10.1016/S0939-6411(00)00076-X

5. Breitenbach J. Melt extrusion: from process to drug delivery technology. Eur J Pharm Sci 2002; 54(2): 107-11.

6. Dannenfelser R, He H, Joshi Y, Bateman S, Serajuddin ATM. Development of clinical dosage forms for a poorly water soluble drug I: Application of polyethylene glycol-polysorbate 80 solid dispersion carrier system. J Pharm Sci 2004; 93: 1165-1175. http://dx.doi. org/10.1002/jps.20044 PMid:15067693

7. Wang L, Cui FD, Hyase T, Sunada H. Preparation and evaluation of solid dispersion for nitrendipine-carbopol and nitrendipine-hpmc systems using a twin screw extruder. Chem Pharm Bull 2005; 53(10): 1240-1245. http://dx.doi.org/10.1248/cpb.53.1240
8. Serajuddin ATM, Sheen PC, Augustine MA. Improved dissolution of a poorly water-soluble drug from solid dispersion in polyethylene glycol: polysorbate 80 mixtures. J Pharm Sci 2006; 79:b463-464. http://dx. doi.org/10.1002/jps.2600790524

9. Pouton $\mathrm{CW}$. Formulation of poorly water-soluble drugs for oral administration: Physicochemical and physiological issues and lipid formulation classification system. Eur J Pharm Sci 2006; 29(3-4): 278287. A http://dx.doi.org/10.1016/j.ejps.2006.04.016 PMid:16815001

10. Jahan ST, Khan MSR, Moniruzzaman M, Rahaman MR, Sadat SMA, Jalil R. Enhancement of dissolution profile for oral delivery of Fexofenadine hydrochloride by solid dispersion (solvent evaporation) technique. Am J Sci Ind Res 2011; 2(1): 112-115.

11. Raval AJ, Patel MM. Preparation and Characterization of Nanoparticles for Solubility and Dissolution Rate Enhancement of Meloxican. Int. R. J. of Pharmaceut 2011; 1(2): 42-49.

12. Kolivoda A, Fischbach M, Kleinebudde P. Application of mixtures of polymeric carriers for dissolution enhancement of fenfibrate using hotmelt extrusion. Int J Pharmaceut 2012; 429(1-2): 58-68. http://dx .doi.org/10.1016/j.ijpharm.2012.03.009 PMid:22440149

13. Reenan T, Vandana D. Solid dispersion: A fruitful approach for improving the solubility and dissolution rate of poorly water soluble drugs. J Drug Del Therapeutics 2012; 2: 5-14.

14. Alam Ma, Aki R, Al Jenoobi FI, Al Mohizea AM. Solid dispersions: a strategy for poorly aqueous soluble drugs and technology updates. Expert Opin Drug Del 2012; 9(11): 1419-1440.

\section{Cite this article as:}

Sarkar Sushanta, Islam Md. Saiful. Solubility improvement of poorly watersoluble drug fenofibrate by solid dispersion in polyethylene glycol-hydroxy propyle methyle cellulose mixture. Int. Res. J. Pharm. 2013; 4(11):33-36 http://dx.doi.org/10.7897/2230-8407.041108 